14. McCloskey R 1979 Corynebacterium species (including diphtheria). In: Mandell GL (ed) Principles and Practice of Infectious Diseases. Wiley, New York

15. Opie LH 1968 Metabolism of the heart in health and disease. Am Heart J 76:685

16. Pappenheimer AM 1977 Diphtheria toxin. Annu Rev Biochem 46:69

17. Rebouche CJ, Engel AG 1980 Tissue distribution of carnitine. Biosynthetic enzymes in man. Biochim Biophys Acta 630:22

18. Tripp M, Katchel M, Peters H, Gilbert E, Arya S, Hodach R, Shug AL 1981
Systemic carnitine deficiency presented as familial endocardial fibroelastosis. N Engl J Med 305:385

19. Wittels B, Bressler R 1965 The effect of diphtheria toxin on carnitine metab olism in the heart. Biochim Biophys Acta 104:39

20. Wurzburg U, Henrich N, Orth HD, Lang H, Prellwitz W, Neumeier D, Knedel M, Rick W 1977 Quantitative determination of creatine kinase isoenzyme catalytic concentrations in serum using immunological methods. J Clin Chem Clin Biochem 15:131

\title{
Regulation of Upper Airway Maintaining Muscles during Progressive Asphyxia
}

\author{
O. P. MATHEW, B. T. THACH, Y. K. ABU-OSBA, R. T. BROUILLETTE, AND J. L. ROBERTS \\ Department of Pediatrics, University of Texas Medical Branch, Galveston, Texas and Washington University \\ School of Medicine, St. Louis Children's Hospital, St. Louis, Missouri 63110
}

\section{Summary}

The electromyographic activity of an upper airway muscle (genioglossus, GG) and the diaphragm were studied in 10 adult and three young anesthetized rabbits during progressive asphyxia induced by airway occlusion. Results were similar for both age groups. Peak inspiratory activity of GG muscle increased more than that of the diaphragm during both the hyperpnea and gasping $(P<0.05)$. The increase in GG activity during gasping was not significantly different from that during hyperpnea even though an important stimulus, arousal, was absent during gasping. During end stage asphyxia, as the strength of gasps grew weaker, the rate of loss of GG muscle activity was greater than that of the diaphragm. However, GG activity remained greater than that of the diaphragm at the time of the last spontaneous gasp. As asphyxia progressed, inspiratory duration and the inspiratory contour of integrated electromyogram activity of both muscles changed. These data indicate differences in the control mechanism of the genioglossus and diaphragm during acute severe asphyxia. Increased upper airway muscle activity seen during gasping should help preserve upper airway patency and facilitate autoresuscitation by gasping. These observations of coordinated changes in timing and activity of two functionally different respiratory muscles support the concept that gasping is a highly organized function of the respiratory centers.

\section{Abbreviations}

DIA, diaphragm

EMG, electromyogram

GG, genioglossus

Recent observations suggest that pulmonary ventilation requires the coordination of thoracic and upper airway respiratory muscles. Contraction of upper airway dilating muscles prevents

Received November 29, 1983.

Supported in part by grants from National Institutes of Health, HD 10993 and from the American Lung Association.

Correspondence should be addressed to Dr. O. P. Mathew, Perinatal Pediatrics, University of Texas Medical Branch, Galveston, TX 77550. airway closure by counteracting the airway constricting effects of the negative pressure $(1,8,10,16,22)$. When the activity of the airway dilating muscles is depressed relative to that of the thoracic muscles, as occurs during general anesthesia (17), the normal balance is upset and airway collapse can occur. Upper airway obstruction is believed to prolong an apneic spell when asphyxia depresses upper airway muscle activity, presumably by the loss or delay of the arousal response $(18,25)$. Lacking direct observations to support or refute this theory, we have studied the inspiratory time course and peak electromyographic activity of an airway dilating muscle, the genioglossus, and an inspiratory pressure generating muscle, the diaphragm, during progressive asphyxia in an animal model. In these studies we have paid particular attention to the terminal events during asphyxia when failure in the strength of respiratory efforts begins to occur.

\section{MATERIALS AND METHODS}

Ten adult New Zealand White rabbits (weight, $1.5-4 \mathrm{~kg}$ ), anesthetized with pentobarbital (25-40 mg intraperitoneally) and three 4-week-old rabbits (weight, $0.5-0.6 \mathrm{~kg}$ ) anesthetized with urethane $(0.65-1.0 \mathrm{~g} / \mathrm{kg})$ were studied. The animals were studied supine with the head fixed at a $90^{\circ}$ angle to the spine. The rectal temperature was maintained between 38 and $39^{\circ} \mathrm{C}$ with an overhead lamp. Fine bipolar wire electrodes (38-gauge, Isomid Beldon) were used to record diaphragm (DIA) and genioglossus (GG) electromyograms (EMG). The diaphragmatic electrodes were inserted through a subcostal incision which was subsequently closed. An anterior midcervical incision was made through the skin and superficial fascia to expose the myelohyoid muscle and the GG electrodes were inserted through the myelohyoid as reported previously (1). EMG signals were amplified (Grass P15 AC preamplifier) filtered, rectified, and integrated. Animals breathed spontaneously through a tracheal cannula. After recording control activity of DIA and GG, the tracheal airway was occluded. Occurrence of arousal, onset of primary apnea, and the onset of gasping were noted on the record. Arousal was said to occur if there were spontaneous body movements, changes in tone of muscles of the head and neck, or eye movements. Direct and integrated EMGs as well as tracheal pressures were recorded on a polygraph. In three of 10 adult rabbits, 
tracheal occlusion was released after the onset of gasping to determine whether they can recover spontaneously once gasping occurred.

\section{DATA ANALYSIS}

GG and DIA activities were calculated from the integrated EMG. Peak inspiratory activity of DIA and GG immediately preceding airway occlusion served as control. Peak inspiratory activity during the hyperpneic and gasping phases was calculated and expressed as percentage of control. In addition, the peak activity of the last three gasps was also analyzed in the same manner. Values were compared using the paired $t$ test and a $P$ value less than 0.05 was considered significant.

\section{RESULTS}

Arousal during airway occlusion. All four stages (hyperpnea, primary apnea, gasping, and secondary apnea) described previously were seen during airway occlusion. As asphyxia progressed, a marked increase in spontaneous body movements such as leg extension and hyperextension of the neck occurred. As primary apnea began, all spontaneous movements other than those immediately related to gasps, were absent. Thus, arousal was seen

$0.5 \vee[$

GG EMG

$2.5 \vee[$

DIA EMG
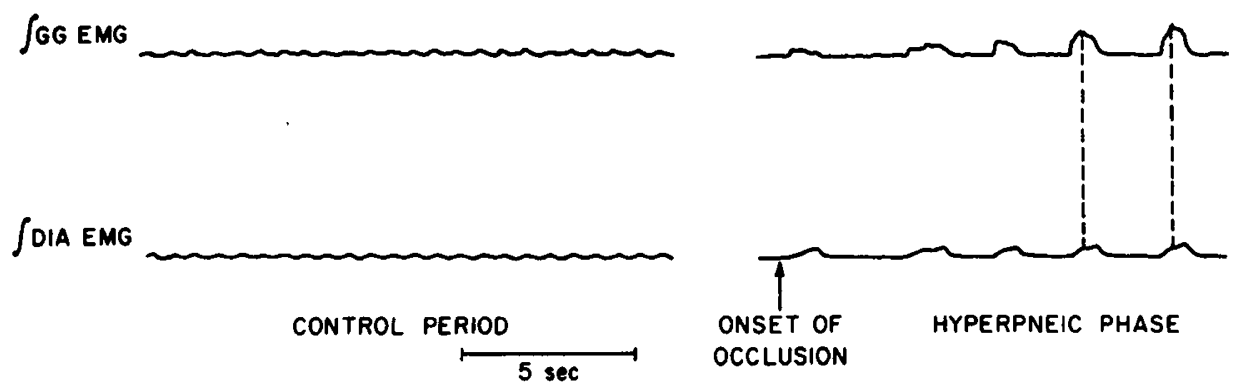

Fig. 1. GG and DIA activity during control period and immediately following airway occlusion in an adult rabbit. Left panel shows baseline activity during the control period prior to airway occlusion. Right panel shows increased GG and DIA activity following airway occlusion. Progressive increase in peak activity during airway occlusion can be seen. Note the plateau-shaped contour of the integrated GG EMG during early occluded inspiratory efforts. GG peaks prior to the DIA (timing of the peak in GG is shown by dotted lines).
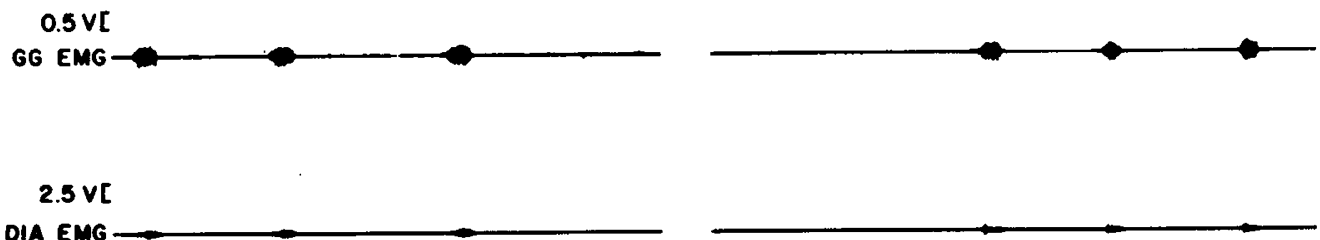

DIA EMG
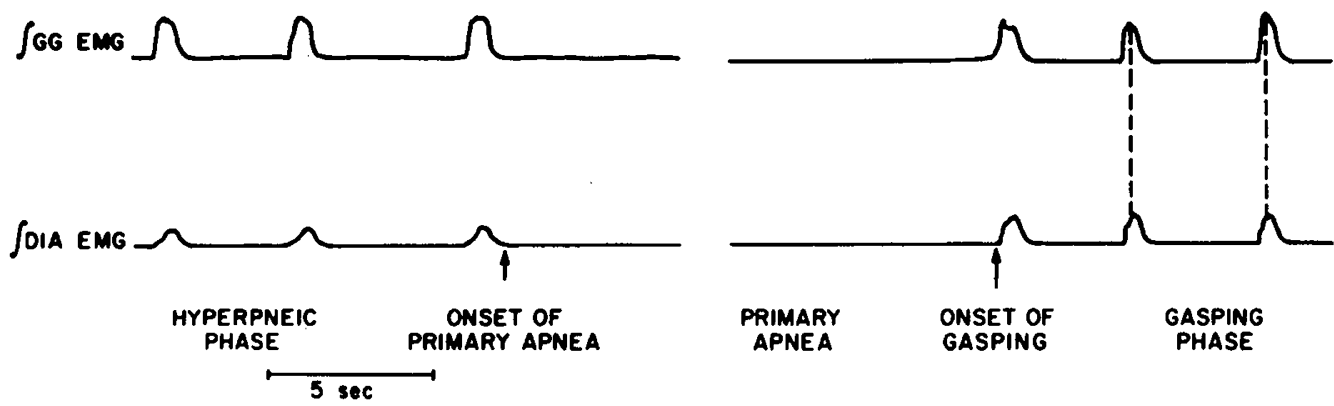

Fig. 2. GG and DIA activity during late in hyperpnea and early in gasping in the same animal as in Figure 1. Note that increased GG and DIA activity are seen in both phases. Also note the markedly decreased inspiratory duration of gasps compared to the initial occluded breaths in hyperpnea (Fig. 1) ( 0.9 vs. $0.3 \mathrm{sec}$ ). Peak inspiratory activity of GG nearly coincides with peak DIA activity as shown by the dotted lines. The GG EMG has become more peaked than plateau. 


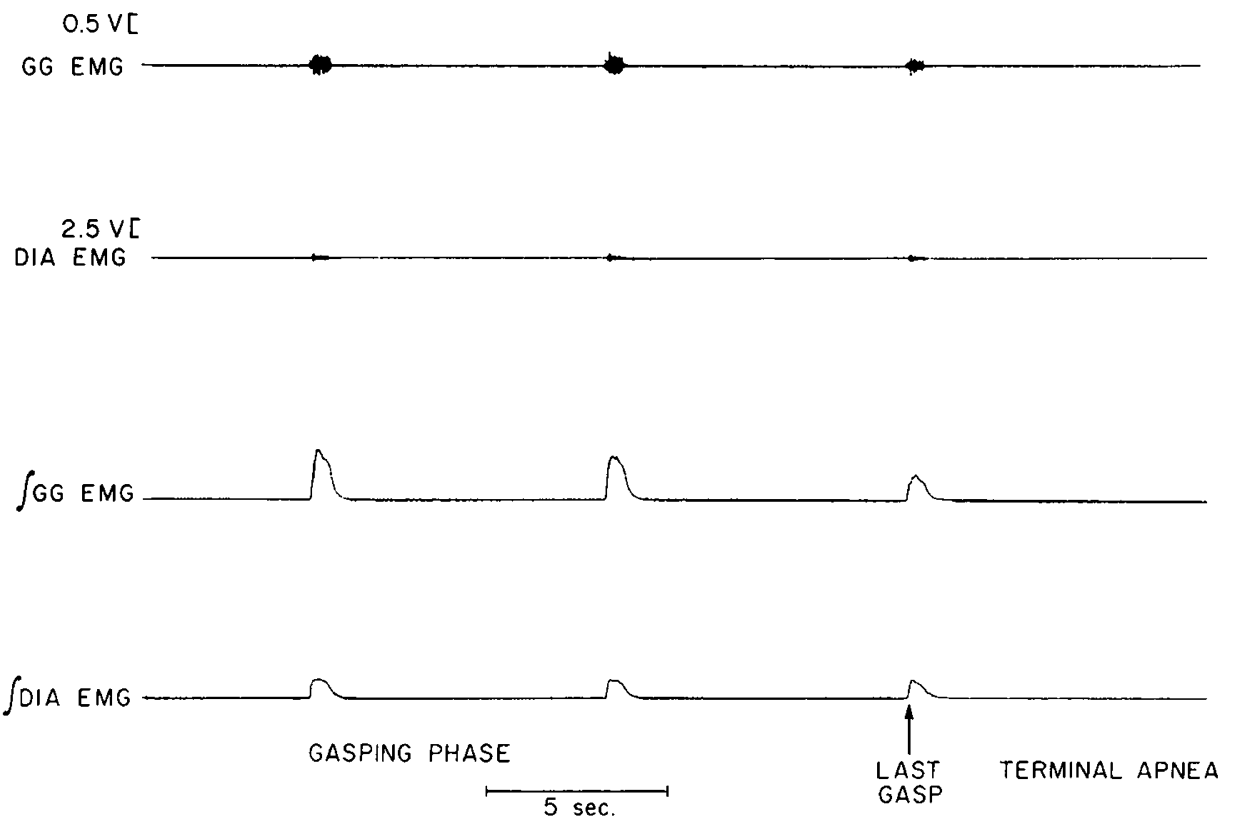

Fig. 3. GG and DIA activity during the last three gasps. Same animal as in Figures 1 and 2. Note that GG activity decreases markedly during these three breaths. The decrease in DIA activity was less marked.

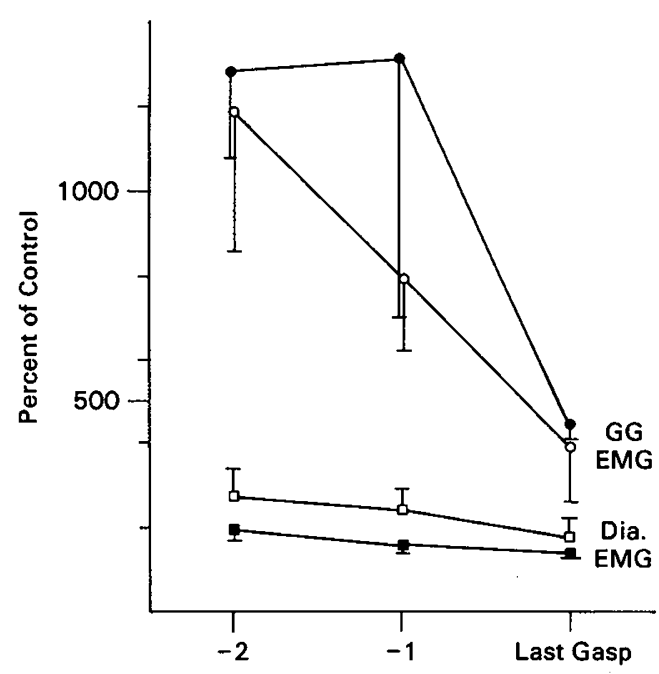

Fig. 4. EMG activity of both GG and DIA (mean and standard error) during the last three gasps $(-2,-1$, and last gasp) are shown. Open symbols, adult animals; filled symbols, young animals; circle, GG activity; square, DIA activity. Both GG and DIA activity decreases. However, in both young and adult animals, the rate of decrease is greater in GG than in DIA.

in all animals during the hyperpneic phase but not during the gasping phase.

Peak activity and inspiratory pattern during hyperpnea and gasping. Peak GG activity during hyperpnea and gasping was increased compared to the control period immediately preceding airway occlusion (Figs. 1 and 2). The increases in GG activity seen during gasping and during the hyperpnea were significantly different $(P<0.005$ and $<0.01$, respectively) from control. GG activity during gasping was not significantly different $(P>0.05)$ from hyperpnea. Peak GG activity remained relatively stable during gasping except towards the end when a decrease in EMG activity was seen (Figs. 3 and 4$)(P<0.05)$.

Peak DIA activity was also increased significantly during both hyperpnea and gasping $(P<0.05$ and $<0.005$, respectively)
Table 1. Peak genioglossus and diaphragm activity during hyperpnea and gasping. GG and DIA activity during these phases are expressed as percentage of their control activity*

\begin{tabular}{|c|c|c|c|c|}
\hline & \multicolumn{2}{|c|}{$\begin{array}{l}\text { Hyperpneic phase } \\
\text { (Arousal present) }\end{array}$} & \multicolumn{2}{|c|}{$\begin{array}{l}\text { Gasping phase } \\
\text { (No arousal) }\end{array}$} \\
\hline & Adult & Newborn & Adult & Newborn \\
\hline $\begin{array}{l}\text { Peak diaphragm } \\
\text { EMG (\% con- } \\
\text { trol) }\end{array}$ & $637 \pm 308$ & $529 \pm 58$ & $402 \pm 133$ & $363 \pm 13$ \\
\hline $\begin{array}{l}\text { Peak genioglossus } \\
\text { EMG (\% con- } \\
\text { trol) }\end{array}$ & $3557 \pm 1701$ & $3767 \pm 505$ & $1766 \pm 512$ & $3533 \pm 52$ \\
\hline
\end{tabular}

(Figs. 1 and 2) when compared to preocclusion values. DIA activity during the hyperpnea was not significantly different from gasping $(P>0.05)$. A decrease in peak DIA activity was seen just before the last gasp (Fig. 4$)(P<0.05)$.

As asphyxia progressed, inspiratory duration (time from onset to peak activity) decreased in both GG and DIA (Figs. 1 and 2). The contour of GG EMG changed from a "plateau" shape to a sharply peaked shape.

Comparison of genioglossus versus diaphragm. Increases in GG activity during hyperpnea and gasping were associated with a simultaneous increase in DIA activity as mentioned above. However, the increase in GG was greater than the increase in DIA (Table 1) $(P<0.05)$. In contrast, in the terminal gasps, both GG and DIA activity showed a decrease in peak EMG activity. The decrease in GG was, however, disproportionately greater compared to the diaphragm (Fig. 4) $(P<0.05)$.

Spontaneous recovery occurred in all three adult rabbits in whom the airway occlusion was released after the onset of gasping.

Results obtained in three young rabbits were similar to that seen in adult animals (Table 1).

\section{DISCUSSION}

In the present studies, following occlusion of the tracheal airway, peak inspiratory activity of the genioglossus muscles 
increased more than that of the diaphragm. This "preferential activation" of these upper airway muscles could originate from several reflex pathways. The stimuli for these reflexes include elevated $\mathrm{PaCO}_{2}$ and reduced $\mathrm{PaO}_{2}(2,26)$, airway mechanoreceptor stimuli $(2,11,24)$, and central nervous system arousal $(2,13$, 16). Thus, the markedly increased activity of the genioglossus muscles during the hyperpneic stage of asphyxia might be explained by the effects of any one of a number of stimuli to the respiratory centers. However, the increased peak activity of these upper airway muscles during the gasping stage is less readily explained. The above mentioned reflex pathways are ineffective during the gasping stage at which time the animal is comatose and shows no evidence of central nervous system arousal. The electroencephalogram is flat, and muscle tone and spontaneous activity are absent $(3,9)$. Furthermore, recent evidence suggests that the respiratory centers are no longer responsive to chemoreceptor stimuli during the gasping stage (19). Finally, it has been established that reflexes from pulmonary airway mechanoreceptors are also abolished during gasping (5). Whether or not reflexes from upper airway mechanoreceptors (11) are active during asphyxial gasping is unclear. However, these reflexes are not relevant to the present findings since airway occlusion was performed at the trachea, and the upper airway was bypassed. In view of the fact that the various reflex pathways known to cause preferential activation of upper airway muscles are believed to be inactive during the gasping stage of asphyxia, the present data suggest that such activation is an intrinsic part of the gasping mechanism.

In the present study, we found that the duration of genioglossus muscle inspiratory activity became shorter as asphyxia progressed and the contour of the electromyogram changed. Similar changes took place in the diaphragm electromyogram. These EMG changes suggest that activity of these muscles is well coordinated to ensure maximum airway dilating effect when the airway constricting pressure is at its peak. The marked change that occurs in the discharge pattern of phrenic nerve motor units during gasping has recently been described (20). These studies of phrenic motor units have suggested marked differences in the respiratory control mechanisms of the gasping and eupneic breathing patterns. The present observations indicate that the changes influencing timing of motor unit discharge during gasping affect the genioglossus and diaphragm similarly.

Although the respiratory activities of genioglossus and diaphragm muscles have a common origin in the medullary respiratory centers, differences in control mechanisms for the two muscles have been previously noted $(1,24,26)$. Additionally, it has frequently been observed that during end stage asphyxia, gasps become progressively more rapid but at the same time weaker $(3,6,9)$. Although this phenomenon has received little attention regarding its mechanism, the progressive loss in power is likely due to decreased output from the respiratory centers rather than decreased contractility of respiratory muscles. This conclusion is based on the fact that respiratory muscles will contract after spontaneous gasps have stopped altogether if their motor nerves are electrically stimulated $(3,23)$. During the terminal stage of gasping, genioglossus muscle control mechanisms are more sensitive to asphyxia than those of the diaphragm. This conclusion is consistent with previous speculations $(18,25)$, and is another example of differences in the respiratory control mechanisms for genioglossus muscle and diaphragm. It should be pointed out, however, that even at the last gasp, peak activity of the genioglossus muscle is greater than that of the diaphragm. Such increased activity of the genioglossus muscle may be adequate for maintaining airway patency.

In past neurophysiologic studies, performed in adult animals, gasping respirations have been viewed as an aberration caused by disintegrating respiratory center function (15). In contrast, in the newborn, gasping has been viewed as an adaptation for survival since it has been shown to be a highly effective mechanism for "autoresuscitation" (3, 7). Recently, Guntheroth and Kawabori (7) have speculated that loss of an efficient gasping reflex during growth and development could be part of a final pathway of events leading to death from an apneic spell in sudden infant death syndrome. This interesting hypothesis remains to be tested. Although maturational changes in the efficacy of gasps have not been clearly defined experimentally, it should be stressed that observations in human patients have documented autoresuscitation by gasping over a wide age range. Peiper (14) and Stevens (21) both observed that young infants could spontaneously recover from sudden, severe asphyxia caused by an apneic spell. In these infants, recovery was attributed to spontaneous gasping respirations like those seen with experimental asphyxia in animals. Similar observations have been made in older children during acute asphyxia secondary to breath-holding spells or sleep apnea $(4,12)$. In the present experiments, results obtained for immature and adult animals were similar. All three adult animals tested rapidly recovered from asphyxial coma when the tracheal airway was reopened. Recovery was the result of their spontaneous gasps, indicating that gasps can still be functionally effective in the adult rabbit. The present observations of coordinated changes in inspiratory timing and peak activity of two respiratory muscles with different functions support the concept that gasping is a complex, highly organized function of the respiratory centers in young and mature rabbits alike.

\section{REFERENCES}

1. Brouillette RT, Thach BT 1979 A neuromuscular mechanism maintaining extrathoracic airway patency. J Appl Physiol 46:772

2. Brouillette RT. Thach BT 1980 Control of genioglossus muscle inspiratory activity. J Appl Physiol 49:801

3. Dawes GS 1968 Foetal and Neonatal Physiology: a Comparative Study of the Changes at Birth. Year Book Medical Publishers, Inc, Chicago

4. Gauk EW, Kidd L. Prichard JS 1963 Mechanism of seizures associated with breathholding spells. N Engl J Med 268:1436

5. Godfrey S 1968 Respiratory and cardiovascular changes during asphyxia and resuscitation of fetal and newborn rabbits. Q J Exp Physiol 53:97

6. Godfrey S 1968 Blood gases during asphyxia and resuscitation of fetal and newborn rabbits. Respir Physiol 4:309

7. Guntheroth WG, Kawabori I 1975 Hypoxic apnea and gasping. J Clin Invest $56: 1371$

8. Hyland RH. Hutcheon MA. Perl A. Bowes G, Anthonisen NR, Zamel N, Phillipson EA 1981 Upper airway occlusion induced by diaphragm pacing for primary alveolar hypoventilation: implications for the pathogenesis of obstructive sleep apnea. Am Rev Respir Dis 124:180

9. Lawson EE. Thach BT 1977 Respiratory patterns during progressive asphyxia in newborn rabbits. J Appl Physiol 43:468

10. Longobardo GS, Gothe B, Goldman MD, Cherniack NS 1982 Sleep apnea considered as a control system instability. Respir Physiol 50:311

11. Mathew OP, Abu-Osba YK, Thach BT 1982 Influence of upper airway pressure changes on genioglossus muscle respiratory activity. J Appl Physiol 52:438

12. Moskowitz MA, Fisher JN, Simpser MD, Strieder DJ 1976 Periodic apnea, exercise hypoventilation and hypothalamic dysfunction. Ann Intern Med $84: 171$

13. Orem J. Dick T, Norris P 1980 Laryngeal and diaphragmatic responses to airway occlusion in sleep and wakefulness. Electroencephalogr Clin Neurophysiol 50:151

14. Peiper A 1973 Cerebral Function in Infancy and Childhood. Consultants Bureau, New York

15. Pitts RF, Magoun HW, Ranson SW 1939 Localization of the medullary respiratory centers in the cat. Am J Physiol 126:673

16. Remmers JE, DeGroot WJ, Sauerland EK, Anch AM 1978 Pathogenesis of upper airway occlusion during sleep. J Appl Physiol 44:931

17. Safar P. Escarraga LA, Chang F 1959 Upper airway obstruction in the unconscious patient. J Appl Physiol 14:760

18. Shannon DC 1980 Pathophysiologic mechanisms causing sleep apnea and hypoventilation in infants. Sleep 3:343

19. St John WM, Knuth KV 1981 A characterization of the respiratory pattern of gasping. J Appl Physiol 50:984

20. St John WM, Bartlett Jr D 1981 Comparison of phrenic motoneuron activity during eupnea and gasping. J Appl Physiol 50:994

21. Stevens LH 1965 Sudden unexplained death in infancy. Am J Dis Child 110:243

22. Tonkin SL, Partridge J, Beach D, Whiteney S 1979 The pharyngeal effect of partial nasal obstruction. Pediatrics 62:261

23. Tschirgi RD, Gerard RW 1947 The carotid-mandibular reflex in acute respiratory failure. Am J Physiol 150:358

24. Van de Graaff WB, Parker D, Van Lunteren E, Strohl K, Mitra J, Cherniack NS 1982 Influence of negative upper airway pressures on respiratory muscle activity and timing. Am Rev Respir Dis 125:219 (abstr)

25. Waggener TB, Frantz III ID, Stark AR, Kronauer RE 1982 Oscillatory breathing patterns leading to apneic spells in infants. J Appl Physiol 52:1288

26. Weiner D, Mitra J, Salamone J, Cherniack NS 1982 Effect of chemical stimuli on nerves supplying upper airway muscles. J Appl Physiol 52:530 